\title{
Polarisation characteristics of optical waves in crystals in the presence of weak optical activity
}

\author{
Adamenko D., Kostyrko M. and Vlokh R. \\ Institute of Physical Optics, 23 Dragomanov St., 79005 Lviv, Ukraine, \\ e-mailvlokh@ifo.lviv.ua
}

Received: 09.04 .2012

Abstract. Basing on the analysis of parameters of the optical waves that propagate in optically uniaxial crystals possessing a weak and ordinary optical activity we have shown that the polarisation characteristics of the outgoing wave should differ for the azimuth of the incoming wave which correspond to the orthogonal eigen components. This fact can be used while measuring the weak optical activity with polarimetric methods.

Keywords: weak optical activity, polarisation

PACS: $78.20 . \mathrm{Ek}$

UDC: 535.56

\section{Introduction}

Optical activity is associated with inhomogeneity of electric displacement field, which leads to the following relationship between the electric induction $D_{i}$ and the electric field $E_{j}$ of optical wave that propagates in a material medium:

$$
D_{i}=\left(\varepsilon_{i j}^{0}+i \delta_{i j l} g_{l k} m_{k}\right) E_{j} .
$$

Here $\varepsilon_{i j}^{0}$ is the tensor of dielectric permittivity in the absence of spatial dispersion, $\delta_{i j l}$ the unit antisymmetric tensor of Levi-Civita, $g_{l k}$ the gyration tensor represented by an axial secondrank tensor, $\vec{m}=(c / \omega) \vec{k}, \vec{k}$ the wave vector, $c$ the speed of light in vacuum, and $\omega$ the angular frequency of the electromagnetic wave. In general, non-symmetric gyration tensor $g_{l k}$ may be decomposed in its symmetric and antisymmetric parts:

$$
g_{l k}=g_{l k}^{s}+g_{l k}^{a s} .
$$

Usually the effect of optical activity is associated with the symmetric part of the gyration tensor (a so-called ordinary optical activity). On the other hand, somewhat different effect is linked to the antisymmetric part of this tensor, which is dual to a polar vector $h_{r}$ (a so-called weak optical activity) [1-5]:

$$
g_{l k}^{a s}=\delta_{l k r} h_{r} .
$$

Taking into account the Neumann symmetry principle, we have $h_{r} \neq 0$ in the point symmetry groups 1, 2, m, mm2, 3, 3m, 4, 4mm, 6, 6mm, $\infty$, and $\infty \mathrm{mm}$. Among these, only the point groups $3 \mathrm{~m}, 4 \mathrm{~mm}, 6 \mathrm{~mm}$ and $\infty \mathrm{mm}$ do not manifest the ordinary optical activity $\left(g_{l k}=0\right)$. Moreover, the following conditions are satisfied for those groups :

$$
g_{l k}=0 ; h_{3} \neq 0 ; h_{1}=h_{2}=0 .
$$

Recent theoretical studies of the weak optical activity have in particular revealed the following:

Ukr. J. Phys. Opt. 2012, V13, №2 
- if the ordinary and weak optical activity are simultaneously present, the polarisation state of light in crystals becomes complex, having a longitudinal-transverse elliptical character [6];

- the weak optical activity should manifest itself as a change in both the refractive indices and optical birefringence [7];

- the Pointing vector of the optical waves that propagate in crystals possessing the weak optical activity has a transverse component, which describes a lateral displacement of the optical beam [8].

Notice that the behaviour of the refractive indices, the optical birefringence and the Pointing vector in the crystalline media revealing the weak optical activity have been analysed for the case of crystals characterised by the point symmetries $3 \mathrm{~m}, 4 \mathrm{~mm}$ and $6 \mathrm{~mm}$ which, in view of the condition given by Eq. (4), seem to be the most suitable for any future experimental studies of the weak optical activity effect.

It is worthwhile that the problems of detection and quantitative studies of the weak optical activity in crystalline media have not yet been solved. In this respect we are to notice that the experimental measurements of the Pointing vector or the refractive indices (eventually, the same refers to the optical birefringence) changed by the weak optical activity can hardly be performed with the aid of existing optical methods, at least due to smallness of the appropriate effects. As a consequence, in the present work we will attempt to develop a theoretical basis necessary for employing standard polarimetric techniques while studying the weak optical activity.

\section{Results of analysis}

We consider an electromagnetic wave propagating through a transparent anisotropic, magnetically disordered medium with accounting of the first-order spatial dispersion phenomena. The following system of equations may be obtained from the Maxwell equations for an electromagnetic wave:

$$
\left(\begin{array}{ccc}
\varepsilon_{11}-m_{2}^{2}-m_{3}^{2} & \varepsilon_{12}+m_{1} m_{2} & \varepsilon_{13}+m_{1} m_{3} \\
\varepsilon_{21}+m_{1} m_{2} & \varepsilon_{22}-m_{1}^{2}-m_{3}^{2} & \varepsilon_{23}+m_{2} m_{3} \\
\varepsilon_{31}+m_{1} m_{3} & \varepsilon_{32}+m_{2} m_{3} & \varepsilon_{33}-m_{1}^{2}-m_{2}^{2}
\end{array}\right)\left(\begin{array}{c}
E_{1} \\
E_{2} \\
E_{3}
\end{array}\right)=0 .
$$

A nontrivial solution of this system is represented by the normal waves

$$
\left(\begin{array}{c}
E_{1} \\
E_{2} \\
E_{3}
\end{array}\right) e\left(t-\frac{(\vec{m} r)}{c}\right)
$$

propagating in a crystal along a certain direction (with $t$ denoting the time variable and $\bar{r}$ the radius vector). These waves correspond to the $m_{i}$ values for which the determinant of Eq. (5) is equal to zero:

$$
\left|\begin{array}{ccc}
\varepsilon_{11}-m_{2}^{2}-m_{3}^{2} & \varepsilon_{12}+m_{1} m_{2} & \varepsilon_{13}+m_{1} m_{3} \\
\varepsilon_{21}+m_{1} m_{2} & \varepsilon_{22}-m_{1}^{2}-m_{3}^{2} & \varepsilon_{23}+m_{2} m_{3} \\
\varepsilon_{31}+m_{1} m_{3} & \varepsilon_{32}+m_{2} m_{3} & \varepsilon_{33}-m_{1}^{2}-m_{2}^{2}
\end{array}\right|=0
$$

\subsection{Crystals belonging to the point symmetry groups $3 \mathrm{~m}, 4 \mathrm{~mm}$ and $6 \mathrm{~mm}$}

For the crystals that belong to the point groups of symmetry $3 \mathrm{~m}, 4 \mathrm{~mm}$ and $6 \mathrm{~mm}$, the dielectric permittivity tensor $\varepsilon_{i j}$ may be represented as 


$$
\varepsilon_{i j}=\left(\begin{array}{ccc}
\varepsilon_{11}^{0} & 0 & i h_{3} m_{1} \\
0 & \varepsilon_{11}^{0} & i h_{3} m_{2} \\
-i h_{3} m_{1} & -i h_{3} m_{2} & \varepsilon_{33}^{0}
\end{array}\right) .
$$

Then Eq. (7) may be rewritten to

$$
\left|\begin{array}{ccc}
\varepsilon_{11}^{0}-m_{2}^{2}-m_{3}^{2} & m_{1} m_{2} & \left(m_{3}+i h_{3}\right) m_{1} \\
m_{1} m_{2} & \varepsilon_{11}^{0}-m_{1}^{2}-m_{3}^{2} & \left(m_{3}+i h_{3}\right) m_{2} \\
\left(m_{3}-i h_{3}\right) m_{1} & \left(m_{3}-i h_{3}\right) m_{2} & \varepsilon_{33}^{0}-m_{1}^{2}-m_{2}^{2}
\end{array}\right|=0 .
$$

Here we consider the case of light propagation along the $x$ axis $\left(m_{2}=m_{3}=0\right)$. For this case the determinant of the matrix given by Eq. (9) becomes

$$
\left|\begin{array}{ccc}
\varepsilon_{11}^{0} & 0 & i h_{3} m_{1} \\
0 & \varepsilon_{11}^{0}-m_{1}^{2} & 0 \\
-i h_{3} m_{1} & 0 & \varepsilon_{33}^{0}-m_{1}^{2}
\end{array}\right|=0 \text {. }
$$

It is easy to prove that the solutions of Eq. (10) correspond to the following $m_{1}$ values:

$$
\left(m_{1}\right)_{1}=\sqrt{\varepsilon_{11}^{0}} ;\left(m_{1}\right)_{2}=\sqrt{\frac{\varepsilon_{11}^{0} \varepsilon_{33}^{0}}{\varepsilon_{11}^{0}+h_{3}^{2}}} .
$$

Inserting the parameters given by Eq. (11) into Eq. (5), one can obtain the relations for the normal waves:

$$
\begin{aligned}
& \left(\begin{array}{l}
0 \\
1 \\
0
\end{array}\right) e^{i \omega\left(t-\frac{1}{c} \sqrt{\varepsilon_{11}^{0}} x\right)} \text { for } m_{1}=\sqrt{\varepsilon_{11}^{0}} \text {, }
\end{aligned}
$$

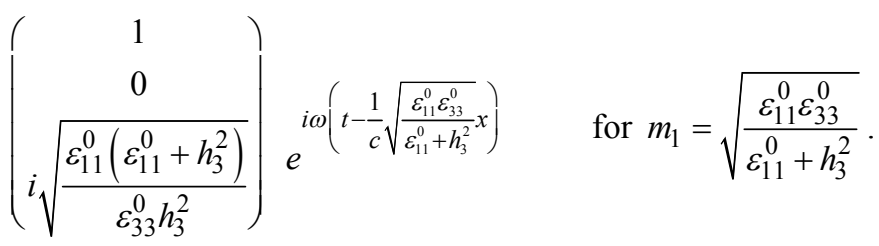

Since an arbitrary electromagnetic wave propagating in crystal along a given direction can be represented as a superposition of the normal waves for this direction, the electromagnetic wave propagating along the $x$-axis can be expressed as

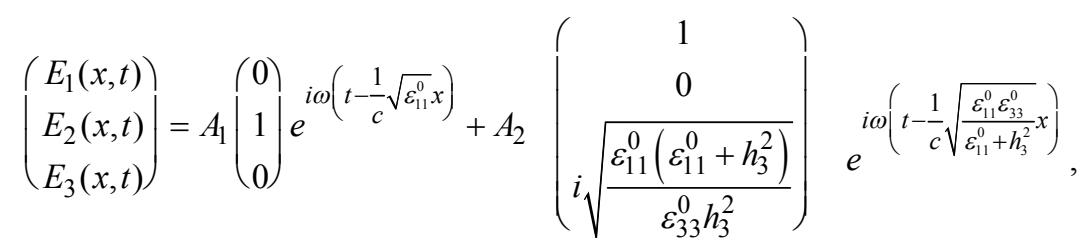

where $A_{1}, A_{2}=$ const. Excluding the time variable from Eq. (14) and considering only the transverse components of the electromagnetic wave, we obtain 


$$
\left(\begin{array}{l}
E_{2}(x) \\
E_{3}(x)
\end{array}\right)=A_{1}\left(\begin{array}{l}
1 \\
0
\end{array}\right) e^{-\frac{i \omega}{c} \sqrt{\varepsilon_{11}^{0}} x}+A_{2}\left(i \sqrt{\frac{\varepsilon_{11}^{0}\left(\varepsilon_{11}^{0}+h_{3}^{2}\right)}{\varepsilon_{33}^{0} h_{3}^{2}}}\right) e^{-\frac{i \omega}{c} \sqrt{\frac{\varepsilon_{11}^{0} \varepsilon_{33}^{0}}{\varepsilon_{11}^{0}+h_{3}^{2}}}} .
$$

Let the input wave at $x=0$ be given by

$$
\left(\begin{array}{l}
E_{2}(0) \\
E_{3}(0)
\end{array}\right)=A_{1}\left(\begin{array}{l}
1 \\
0
\end{array}\right)+A_{2}\left(i \sqrt{\frac{\varepsilon_{11}^{0}\left(\varepsilon_{11}^{0}+h_{3}^{2}\right)}{\varepsilon_{33}^{0} h_{3}^{2}}}\right) .
$$

Then we get

$$
\left(\begin{array}{c}
E_{2}(x) \\
E_{3}(x)
\end{array}\right)=\left(\begin{array}{cc}
e^{-\frac{i \omega}{c} \sqrt{\varepsilon_{11}^{0}} x} & 0 \\
0 & e^{-\frac{i \omega}{c} \sqrt{\frac{\varepsilon_{11}^{0} \varepsilon_{33}^{0}}{\varepsilon_{11}^{0}+h_{3}^{2}}}}
\end{array}\right)\left(\begin{array}{c}
E_{2}(0) \\
E_{3}(0)
\end{array}\right)
$$

When the input light is linearly polarised along the $y$ axis, one can rewrite Eq. (17) as

$$
\left(\begin{array}{l}
E_{2}(x) \\
E_{3}(x)
\end{array}\right)=\left(\begin{array}{cc}
e^{-\frac{i \omega}{c} \sqrt{\varepsilon_{11}^{0}} x} & 0 \\
0 & e^{-\frac{i \omega}{c} \sqrt{\frac{\varepsilon_{11}^{0} \varepsilon_{33}^{0}}{\varepsilon_{11}^{0}+h_{3}^{2}}}}
\end{array}\right)\left(\begin{array}{c}
E_{2}(0) \\
0
\end{array}\right),
$$

or

$$
E_{2}(x)=e^{-\frac{i \omega}{c} \sqrt{\varepsilon_{11}^{0}} x} E_{2}(0)
$$

Quite similarly, when the input light is linearly polarised along the $z$ axis, one can rewrite Eq. (17) as

$$
\left(\begin{array}{c}
E_{2}(x) \\
E_{3}(x)
\end{array}\right)=\left(\begin{array}{cc}
e^{-\frac{i \omega}{c} \sqrt{\varepsilon_{11}^{0}} x} & 0 \\
0 & e^{-\frac{i \omega}{c} \sqrt{\frac{\varepsilon_{11}^{0} \varepsilon_{33}^{0}}{\varepsilon_{11}^{0}+h_{3}^{2}}}}
\end{array}\right)\left(\begin{array}{c}
0 \\
E_{3}(0)
\end{array}\right)
$$

or

$$
E_{3}(x)=e^{-\frac{i \omega}{c} \sqrt{\frac{\varepsilon_{11}^{0} \varepsilon_{33}^{0}}{\varepsilon_{11}^{0}+h_{3}^{2}}} x} E_{3}(0) .
$$

In the both cases, the input linearly polarised light remains linearly polarised after having passed through the crystal.

In other words, the state of light polarisation in the $y z$ plane is not changed in the crystals belonging to the point groups $3 \mathrm{~m}, 4 \mathrm{~mm}$ and $6 \mathrm{~mm}$, whenever the linearly polarised light passes through the crystalline medium under the condition $\vec{k} \| x$. This fact renders impossible any studies for the weak optical activity in crystals, based on the polarimetric techniques.

\subsection{Crystals belonging to the point symmetry groups 3,4 and 6}

The crystals that belong to the symmetry groups 3,4 , and 6 possess both the weak and ordinary optical activities. The dielectric permittivity tensor $\varepsilon_{i j}$ is as follows: 


$$
\varepsilon_{i j}=\left(\begin{array}{ccc}
\varepsilon_{11}^{0} & i g_{33} m_{3} & -i\left(g_{11} m_{2}-h_{3} m_{1}\right) \\
-i g_{33} m_{3} & \varepsilon_{11}^{0} & i\left(g_{11} m_{1}+h_{3} m_{2}\right) \\
i\left(g_{11} m_{2}-h_{3} m_{1}\right) & -i\left(g_{11} m_{1}+h_{3} m_{2}\right) & \varepsilon_{33}^{0}
\end{array}\right) .
$$

Then Eq. (7) may be written as

$$
\left|\begin{array}{ccc}
\varepsilon_{11}^{0}-m_{2}^{2}-m_{3}^{2} & m_{1} m_{2}+i g_{33} m_{3} & m_{1} m_{3}-i\left(g_{11} m_{2}-h_{3} m_{1}\right) \\
m_{1} m_{2}-i g_{33} m_{3} & \varepsilon_{11}^{0}-m_{1}^{2}-m_{3}^{2} & m_{2} m_{3}+i\left(g_{11} m_{1}+h_{3} m_{2}\right) \\
m_{1} m_{3}+i\left(g_{11} m_{2}-h_{3} m_{1}\right) & m_{2} m_{3}-i\left(g_{11} m_{1}+h_{3} m_{2}\right) & \varepsilon_{33}^{0}-m_{1}^{2}-m_{2}^{2}
\end{array}\right|=0 .
$$

Let us consider again the same case of light propagation along the $x$ axis $\left(m_{2}=m_{3}=0\right)$, for which Eq. (23) becomes

$$
\left|\begin{array}{ccc}
\varepsilon_{11}^{0} & 0 & i h_{3} m_{1} \\
0 & \varepsilon_{11}^{0}-m_{1}^{2} & i g_{11} m_{1} \\
-i h_{3} m_{1} & -i g_{11} m_{1} & \varepsilon_{33}^{0}-m_{1}^{2}
\end{array}\right|=0
$$

The solutions of Eq. (24) are given by the following $m_{1}$ values:

$$
\begin{aligned}
& \left(m_{1}\right)_{1}=M_{1}=\sqrt{\frac{\varepsilon_{11}^{0}\left[\varepsilon_{11}^{0}+\varepsilon_{33}^{0}+g_{11}^{2}+h_{3}^{2}+\sqrt{\left(\varepsilon_{11}^{0}+\varepsilon_{33}^{0}+g_{11}^{2}+h_{3}^{2}\right)^{2}-4\left(\varepsilon_{11}^{0}+h_{3}^{2}\right) \varepsilon_{33}^{0}}\right]}{2\left(\varepsilon_{11}^{0}+h_{3}^{2}\right)},} \\
& \left(m_{1}\right)_{2}=M_{2}=\sqrt{\frac{\varepsilon_{11}^{0}\left[\varepsilon_{11}^{0}+\varepsilon_{33}^{0}+g_{11}^{2}+h_{3}^{2}-\sqrt{\left.\left(\varepsilon_{11}^{0}+\varepsilon_{33}^{0}+g_{11}^{2}+h_{3}^{2}\right)^{2}-4\left(\varepsilon_{11}^{0}+h_{3}^{2}\right) \varepsilon_{33}^{0}\right]}\right.}{2\left(\varepsilon_{11}^{0}+h_{3}^{2}\right)} .} .
\end{aligned}
$$

Inserting the parameters given by Eqs. (25) and (26) into Eq. (5), one can obtain the relations for the normal waves:

$$
\left(\begin{array}{c}
-\frac{i h_{3} M}{\varepsilon_{11}^{0}} \\
-\frac{i g_{11} M}{\varepsilon_{11}^{0}-M^{2}} \\
1
\end{array}\right) e^{i \omega\left(t-\frac{M}{c} x\right)}
$$

where $M=M_{1}, M_{2}$. Hence, we get the following relation for the electromagnetic wave propagating along the $x$ axis:

$$
\left(\begin{array}{l}
\left.E_{1}(x, t)\right) \\
E_{2}(x, t) \\
E_{3}(x, t)
\end{array}\right)=A_{1}\left(\begin{array}{c}
-\frac{i h_{3} M_{1}}{\varepsilon_{11}^{0}} \\
-\frac{i g_{11} M_{1}}{\varepsilon_{11}^{0}-M_{1}^{2}} \\
1
\end{array}\right) e^{i \omega\left(t-\frac{M_{1}}{c} x\right)}+A_{2}\left(\begin{array}{c}
-\frac{i h_{3} M_{2}}{\varepsilon_{11}^{0}} \\
-\frac{i g_{11} M_{2}}{\varepsilon_{11}^{0}-M_{2}^{2}} \\
1
\end{array}\right) e^{i \omega\left(t-\frac{M_{2}}{c} x\right)},
$$

where $A_{1}, A_{2}=$ const. Dropping the time variable and considering only the transverse components of the electromagnetic wave, we rewrite Eq. (28) as 


$$
\left(\begin{array}{c}
E_{2}(x) \\
E_{3}(x)
\end{array}\right)=A_{1}\left(\begin{array}{c}
-\frac{i g_{11} M_{1}}{\varepsilon_{11}^{0}-M_{1}^{2}} \\
1
\end{array}\right) e^{-i \frac{\omega}{c} M_{1} x}+A_{2}\left(\begin{array}{c}
-\frac{i g_{11} M_{2}}{\varepsilon_{11}^{0}-M_{2}^{2}} \\
1
\end{array}\right) e^{-i \frac{\omega}{c} M_{2} x} .
$$

In particular, at $x=0$ we have

$$
\left(\begin{array}{c}
E_{2}(0) \\
E_{3}(0)
\end{array}\right)=A_{1}\left(\begin{array}{c}
-\frac{i g_{11} M_{1}}{\varepsilon_{11}^{0}-M_{1}^{2}} \\
1
\end{array}\right)+A_{2}\left(\begin{array}{c}
-\frac{i g_{11} M_{2}}{\varepsilon_{11}^{0}-M_{2}^{2}} \\
1
\end{array}\right),
$$

and so the relation

$$
\left(\begin{array}{l}
E_{2}(x) \\
E_{3}(x)
\end{array}\right)=\left(\begin{array}{ll}
T_{22} & T_{23} \\
T_{32} & T_{33}
\end{array}\right)\left(\begin{array}{l}
E_{2}(0) \\
E_{3}(0)
\end{array}\right),
$$

holds true, with the parameters

$$
\begin{gathered}
T_{22}=\frac{M_{1}\left(\varepsilon_{11}^{0}-M_{2}^{2}\right) e^{-i \frac{\omega}{c} M_{1} x}-M_{2}\left(\varepsilon_{11}^{0}-M_{1}^{2}\right) e^{-i \frac{\omega}{c} M_{2} x}}{M_{1}\left(\varepsilon_{11}^{0}-M_{2}^{2}\right)-M_{2}\left(\varepsilon_{11}^{0}-M_{1}^{2}\right)}, \\
T_{23}=T_{22}-\frac{i g_{11} M_{1}}{\varepsilon_{11}^{0}-M_{1}^{2}} e^{-i \frac{\omega}{c} M_{1} x}, \\
T_{32}=\frac{i\left(\varepsilon_{11}^{0}-M_{1}^{2}\right)\left(\varepsilon_{11}^{0}-M_{2}^{2}\right)}{g_{11}\left(M_{1}\left(\varepsilon_{11}^{0}-M_{2}^{2}\right)-M_{2}\left(\varepsilon_{11}^{0}-M_{1}^{2}\right)\right)}\left(e^{-i \frac{\omega}{c} M_{1} x}-e^{-i \frac{\omega}{c} M_{2} x}\right), \\
T_{33}=T_{32}+e^{-i \frac{\omega}{c} M_{1} x}
\end{gathered}
$$

being components of the appropriate Jones matrix.

When the input light is linearly polarised along the $y$ axis, we obtain

$$
\left\{\begin{array}{l}
E_{2}(x)=T_{22} E_{2}(0) \\
E_{3}(x)=T_{32} E_{2}(0)
\end{array}\right.
$$

Then the polarisation ratio $\chi_{2}$ may be easily found as

$$
\chi_{2}=\frac{E_{2}(x)}{E_{3}(x)}=\frac{T_{22}}{T_{32}} .
$$

When the input light is linearly polarised along the $z$ axis, we have

$$
\left\{\begin{array}{l}
E_{2}(x)=T_{23} E_{3}(0) \\
E_{3}(x)=T_{33} E_{3}(0)
\end{array} .\right.
$$

The corresponding polarisation ratio $\chi_{3}$ is given by

$$
\chi_{3}=\frac{E_{2}(x)}{E_{3}(x)}=\frac{T_{23}}{T_{33}}=\frac{T_{22}-\frac{i g_{11} M_{1}}{\varepsilon_{11}^{0}-M_{1}^{2}} e^{-i \frac{\omega}{c} M_{1} x}}{T_{32}+e^{-i \frac{\omega}{c} M_{1} x}} \neq \chi_{2} .
$$

It is known that the polarisation ratio $\chi$ is related to the ellipticity $R$ and the azimuth $\beta$ of the polarisation ellipse as follows: 


$$
\begin{gathered}
R=\frac{1+|\chi|^{2}-\sqrt{(1+|\chi|)^{2}+\left(\chi-\chi^{*}\right)^{2}}}{1+|\chi|^{2}+\sqrt{(1+|\chi|)^{2}+\left(\chi-\chi^{*}\right)^{2}}}, \\
\beta=\frac{1}{2} \operatorname{arctg} \frac{\chi+\chi^{*}}{1-|\chi|^{2}} .
\end{gathered}
$$

Therefore, the input linearly polarised light becomes elliptically polarised after having passed through the crystals that belong to the point groups of symmetry 3,4 and 6 under the condition $\vec{k} \| x$. Moreover, the parameters characterising the output polarised light differ for different input polarisations. Since the parameters $R$ and $\beta$ can, in principle, be easily determined with the polarimetric techniques, the weak optical activity in the crystals of the point groups 3,4 and 6 can be detected and measured quantitatively using these techniques.

\section{Conclusion}

We have shown that, for the crystals belonging to the point symmetry groups $3 \mathrm{~m}, 4 \mathrm{~mm}$ and $6 \mathrm{~mm}$, the polarisation state in the $y z$ plane experiences no changes whenever the input linearly polarised light passes through the crystalline medium in the case of $\vec{k} \| x$. This does not enable studies of the weak optical activity using the standard polarimetric methods. On the other hand, the input linearly polarised light becomes elliptically polarised after having passed through the crystals that belong to the point symmetry groups 3,4 and 6 , under the same additional condition $\vec{k} \| x$. As a matter of fact, the parameters of the output polarised light depend on the azimuth of linear polarisation of the light entering the crystal. The latter can be used while studying the weak optical activity, e.g., with the aid of the polarimetric methods.

\section{References}

1. Fedorov F I, 1959. On the theory of optical activity of crystals. 2. Crystals of cubic system and polar classes of medium-symmetry systems. Opt. Spektr. 6: 377-383.

2. Ivchenko E I, Permogorov S A and Sel'kin A V, 1978. Reflection of light with a change of polarization state from the real crystal boundary. Solid State Commun. 28: 345-348.

3. Ivchenko E L, Pevtsov A B and Sel'kin A V, 1981. Effects of optical activity on exciton luminescence of CdS. Solid State Commun. 39: 453-455.

4. Zil'bershtein A Kh and Solov'ev L E, 1998. Reflection of light with the change of polarization state from real crystal boundary. Opt. Spectr. 84: 549-552.

5. Lalov I J and Kojouharova N A, 2000. Reflection of electromagnetic waves at the boundary of optically active media. Bulgarian J. Phys. 27: 66-71.

6. Zapeka B, Kostyrko M and Vlokh R, 2010. Coexistence of ordinary gyration and weak optical activity in crystals: eigen waves of a new type. Ukr. J. Phys. Opt. 11: 119-125.

7. Zapeka B, Kostyrko M and Vlokh R, 2010. On the light refraction and polarization in the presence of weak optical activity. Ukr. J. Phys. Opt. 11: 68-73.

8. Adamenko D, Kostyrko M and Vlokh R, 2011. On the behavior of Pointing vector in material media with weak optical activity. Ukr. J. Phys. Opt. 12: 143-149.

Adamenko D., Kostyrko M. and Vlokh R., 2012. Polarisation characteristics of optical waves in crystals in the presence of weak optical activity. Ukr.J.Phys.Opt. 13: $67-73$.

Анотація. На основі аналізу поляризаційних параметрів оптичних хвиль, що поширюються крізь одновісні кристали, які володіють слабкою і звичайною оптичною активністю, показано, що характеристики поляризації хвилі на виході відрізнятимуться для азимута вхідної хвилі, який відповідає ортогональним власним компонентам. Даний факт можна використати у вимірюваннях слабкої оптичної активності за поляриметричними методами. 